\title{
Validity of Sphenoid Ostium in Relation to Posterior Wall of Maxillary Sinus by Computed Tomography \\ Tarek Abdelmoaty Omran ${ }^{1}$, Sameh Mohammad Hosny ${ }^{1}$, Magdy Kamel Abdelkmaksod ${ }^{1}$, Hend El Sayed Koraim*1, Mohamed Ibrahim Amin², Mohamed Ahmed Alshawadfy ${ }^{1}$ \\ Departments of ${ }^{1}$ Otorhinolaryngology and ${ }^{2}$ Radiodiagnosis, Faculty of Medicine, Zagazig University, Egypt \\ *Corresponding Author: Hend El Sayed Koraim, Email: hndkrym054@gmail.com
}

\begin{abstract}
Background: Sphenoid sinus is the most inaccessible paranasal sinus, enclosed within the sphenoid bone and intimately related to numerous vital neural and vascular structures. Anatomic variation of the sphenoid sinus is well documented and may complicate surgery in such a place.

Objective: To assess the reliability of preoperative computed tomography (CT) in determination of the distance between the posterior wall of maxillary sinus (PWMS) and the sphenoid sinus ostium (SSO) in the coronal plane.

Patients and methods: This was a prospective study of the distance between the SSO and the PWMS in the coronal plane, which was measured both radiologically in preoperative CT and intraoperatively during endoscopic sinus surgery for patients in need for middle meatal antrostomy and sphenoid sinusotomy at the same side. Distances obtained by both techniques have been tested for the degree of correlation. Number of the included patients was 25 .

Results: Forty four nasal sides were included in the study. The mean distance between the sphenoid sinus ostium and posterior wall of maxillary sinus in the coronal plane was $6.6 \pm 1.8 \mathrm{~mm}$ when measured by preoperative CT and $6.9 \pm$ $1.9 \mathrm{~mm}$ when measured directly during surgery. No significant difference was found between the mean distances measured by both techniques $(\mathrm{p}=0.246)$ with good agreement between them $(\mathrm{r}=0.864)$.

Conclusion: Preoperative CT may be a reliable tool to preoperatively determine the difference in depth between the PWMS and the SSO.
\end{abstract}

Keywords: Computed tomography, Posterior wall of maxillary sinus, Sphenoid ostium.

\section{INTRODUCTION}

The sphenoid sinus is related to important vascular and neural structures including the internal carotid artery, cavernous sinus, optic nerve, trigeminal nerve, vidian nerve and pituitary gland ${ }^{(1,2)}$. Also, the position of the sinus at skull base makes it a suitable route to approach the anterior and middle cranial fossae ${ }^{(3,4)}$. So, understanding of the anatomy of the sinus region is essential for ENT and neuro surgeons to avoid major complications during endoscopic sinus surgery (ESS) and skull base surgery.

Although the position of the sphenoid sinus ostium (SSO) may be affected by variability of pneumatization of the sinus and nearby structures, it is still the safest place and one of the most consistent and principal routes to enter the sphenoid sinus. Therefore identification of the ostium is considered a key step during endoscopic surgery ${ }^{(5)}$. This is especially important for beginners and trainers and for even experienced surgeons in revision surgery and in presence of extensive pathological lesions $^{(\mathbf{6})}$.

Different anatomical landmarks have been reported as references for localization of the SSO including medial orbital floor ${ }^{(\boldsymbol{(})}$, posteroinferior end superior turbinate $^{(7)}$, superior margin of choana ${ }^{(8)}$, nasal spine ${ }^{(9)}$, sphenovomerian suture ${ }^{(\mathbf{1 0})}$ and sphenoid skull base ${ }^{(\mathbf{1 1})}$. Few studies in literature have investigated the posterior wall of maxillary sinus (PWMS) as a landmark to locate the SSO either radiologically or by direct measurement during surgery or cadaveric dissection ${ }^{(\mathbf{1 2 - 1 4})}$.

\section{PATIENTS AND METHODS}

This prospective study has been conducted between September 2020 and July 2021 at otolaryngology Head and Neck Surgery Department, Zagazig University (ZU)

Hospitals. 25 patients for whom endoscopic sinus surgery was planned were screened and those who fulfilled criteria of the study were enrolled. Inclusion criteria were age above 18 years and indicated middle meatal antrostomy and sphenoid sinusotomy at the same side. Patients with expansile sinus lesions, destructive sinus pathologies including malignant tumors, craniofacial anomalies and history of maxillofacial or skull base fractures were excluded from the study.

All enrolled patients were subjected to preoperative full history taking, general examination, endoscopic nasal examination, laboratory investigation and $\mathrm{CT}$ of paranasal sinuses.

\section{Ethical considerations:}

All participants signed informed consent and approval of Institutional Review Board (ZU-IBR \# 6563) was obtained. This work has been carried out in accordance with the Code of Ethics of the World Medical Association (Declaration of Helsinki) for studies involving humans. 
Techniques of measurements:

Radiological measurement:

Preoperative paranasal sinuses CT was done and the distance between SSO and PWMS was measured through curved planner reformatted image so as the 2 sinuses can appear together in same line (Fig.1).

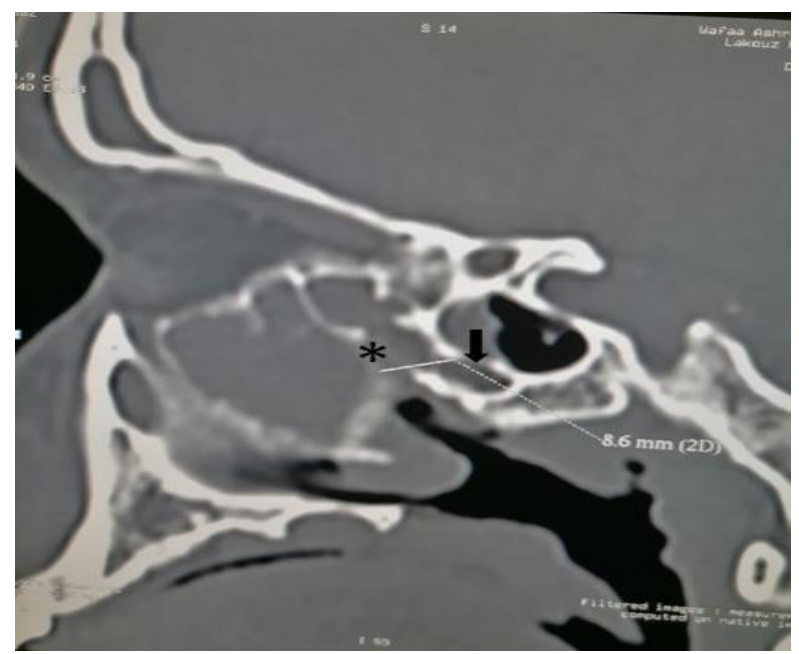

Fig. (1): Radiological measurement of distance between SSO (arrow) and PWMS (asterisk) in reformatted oblique sagittal plane.

\section{Intraoperative measurement:}

After intraoperative identification of the SSO and PWMS, a $4 \mathrm{~mm}$ straight suction tip was used to scale the distances between the base of columella and both of SSO and the deepest point of PWMS. The scaled distances were then measured by a ruler and the difference between both distances was calculated (Fig. 2). The surgeon was blinded with preoperative radiological measurement.
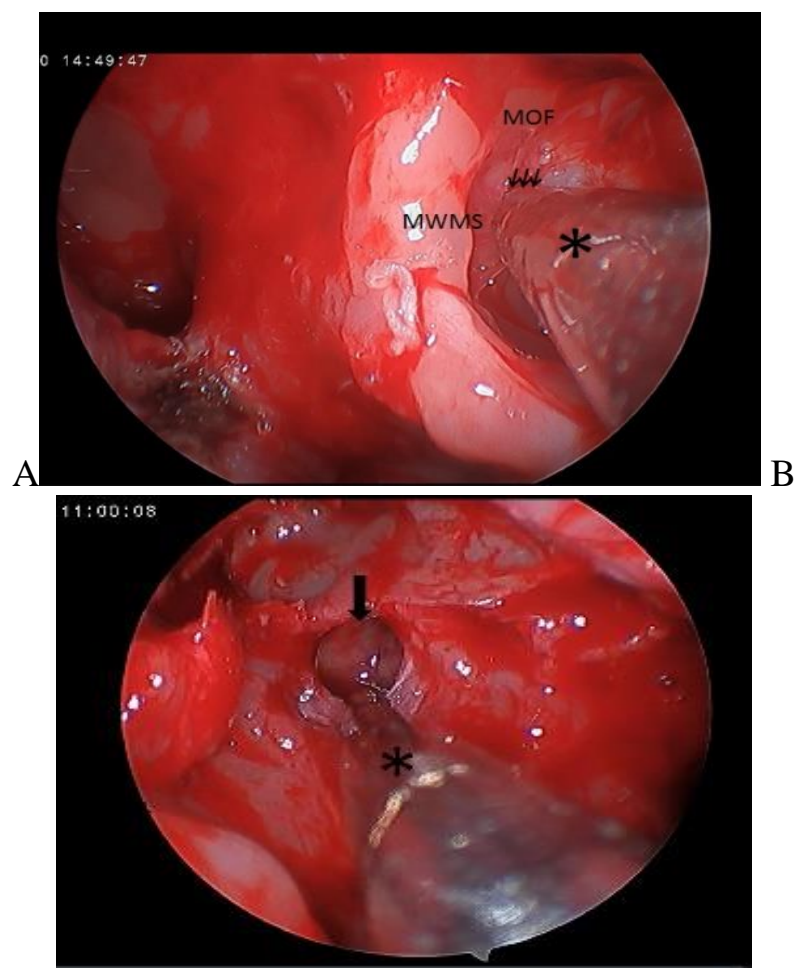

Fig. (2): Intraoperative measurement using $4 \mathrm{~mm}$ suction tip (asterisk). (A) From PWMS (small black arrows) near to medial orbital floor (MOF).
MWMS=medial wall of maxillary inus. (B) From SSO (black arrow).

\section{Statistical analysis}

Statistical data analysis was done using SPSS; version 22. Continuous variables were described as mean \pm SD while categorical variables were described as frequencies and percentages. The t test was used to compare continuous variables. Intraclass correlation was used to detect the degree of agreement between continuous variables. Statistical significance was considered for $\mathrm{p}$ value $<0.5$.

\section{RESULTS}

Most common indication for endoscopic sinus surgery was chronic rhinosinusitis with nasal polyps. Middle meatal antrostomy and sphenoid sinusotomy were done for 19 patients at both side and for 6 patients at one side so the number of studied sides was 44 . The demographic and clinical data of study patients are shown in table 1.

Table (1): Demographic and clinical data of patients

\begin{tabular}{|l|c|}
\hline Patients, no. & 25 \\
operated sides, no. & 44 \\
\hline Age (years) & $18-66$ \\
Range & $42.2 \pm 16.3$ \\
Mean \pm SD & \\
\hline Sex, no (\%) & $9(36 \%)$ \\
Males & $16(64 \%)$ \\
females & \\
\hline Indication of ESS /patient, no (\%) & $19(76 \%)$ \\
CRSwNP & $3(12 \%)$ \\
CRSsNP & $3(12 \%)$ \\
Allergic fungal sinusitis & \\
\hline
\end{tabular}

The mean distance from the base of columella to the SSO was $75.3 \pm 4.4 \mathrm{~mm}$ while the mean distance from base of columella to the PWMS was $68.5 \pm 4.1$ $\mathrm{mm}$. The SSO was found deeper to the PWMS in all sides except one side at which both were at the same level. No statistically significant differences have been found between distances calculated intraoperatively, in the coronal plane, and radiologically (Table 2).

Table (2): The mean distances between PWMS and SSO measured intraoperatively vs radiologically

\begin{tabular}{|c|l|c|}
\hline $\begin{array}{c}\text { Distance between SSO } \\
\text { and PWMS in mm: }\end{array}$ & $\begin{array}{c}\text { Range } \\
\text { (Mean } \pm \\
\text { SD) }\end{array}$ & $\begin{array}{c}\text { P } \\
\text { value }\end{array}$ \\
\hline - Intraoperatively measured & $\begin{array}{l}0-10(6.9 \\
\pm 1.9)\end{array}$ & 0.246 \\
- Radiologically measured & $\begin{array}{l}3.9-9.1 \\
(6.6 \pm 1.8) .\end{array}$ \\
\hline
\end{tabular}

There was a good agreement between intraoperative and radiological measurement techniques in detection of distance between SSO and PWMS (interclass correlation coefficient $=0.864,95 \%$ CI: $0.752-0.925$ ) (Fig. 3). 


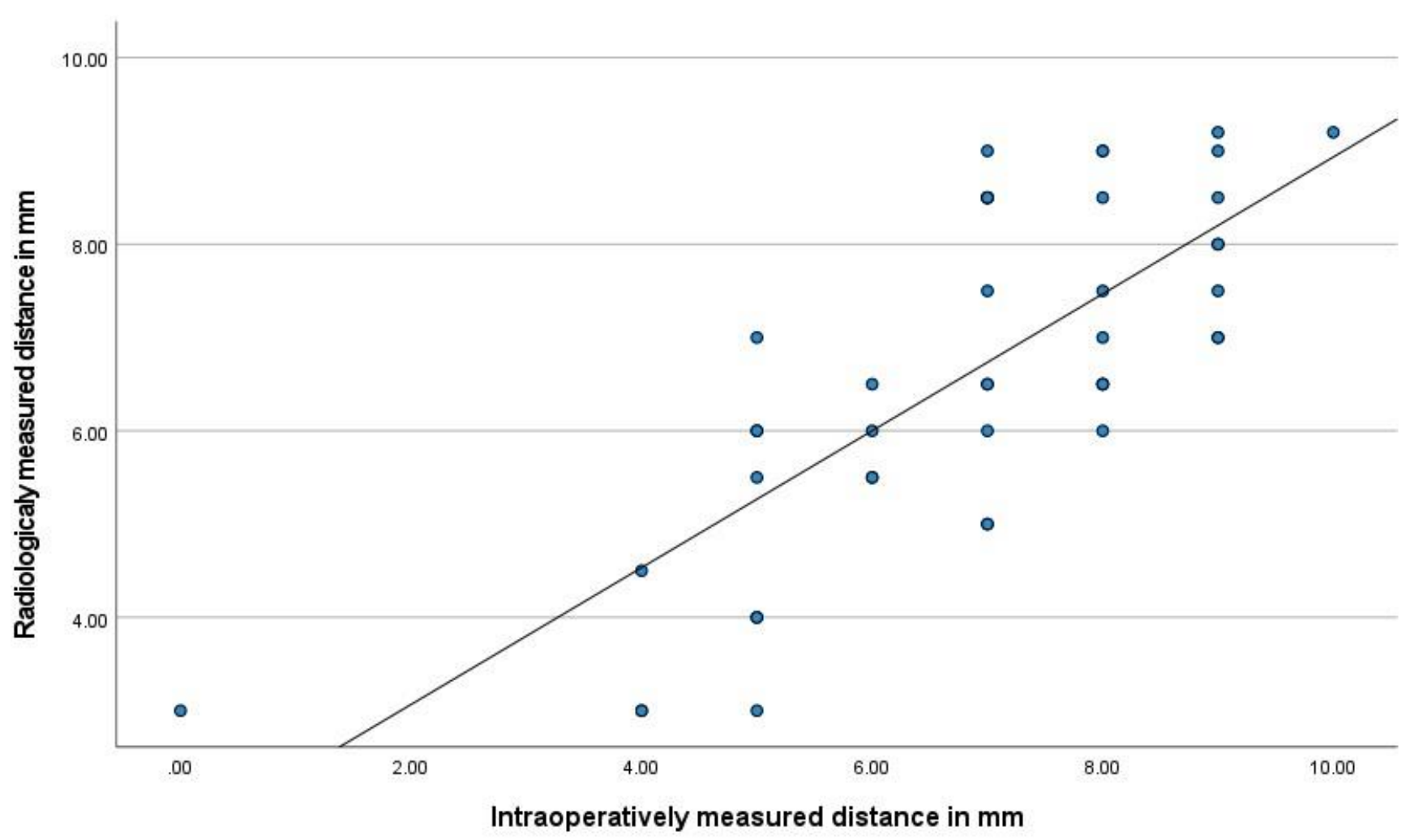

Fig. (3): Scatter plot showing correlation between intraoperatively measured distances and radiologically measured distances between the SSO and PWMS.

\section{DISCUSSION}

In the present study the distance between the PWMS and the SSO in the coronal plane was measured by $\mathrm{CT}$ and intraoperatively during endoscopic sinus surgery (ESS). We found that the mean depth of the PWMS and SSO from the base of columella was $75.3 \pm$ $4.4 \mathrm{~mm}$ and $68.5 \pm 4.1 \mathrm{~mm}$ respectively. The mean distance between SSO and PWMS in the coronal plane was $6.9 \pm 1.9 \mathrm{~mm}$ by intraoperative measurement and $6.6 \pm 1.8 \mathrm{~mm}$ when measured radiologically.

The SSO was found at a level deep to PWMS in all studied sides except one side at which both sites were at the same level. Such findings are consistent with those reported in the cadaveric dissection study of Casiano $^{(6)}$ who applied the same measurement technique used in this study and found a mean of 7.45 $\mathrm{cm}$ and $6.95 \mathrm{~cm}$ for the distances from the base of columella to each of SSO and PWMS respectively. He found also that the anterior face of sphenoid was posterior to the PWMS in all examined sides with an average of 2-4 $\mathrm{mm}$ between them.

Our finding, regarding the difference in depth between the two anatomical sites also agree with the CT based readings of Albahkaly et al. ${ }^{(12)}$ who have reported an average of $7.5 \mathrm{~mm}$ difference in the coronal plane between both sites. A smaller average $(1.5 \pm 1.4$ $\mathrm{mm}$ ) of the intraoperatively measured distance between SSO and PWMS has been reported by Dedhia et al. ${ }^{(13)}$. They have also mentioned that SSO was not always posterior to PWMS but at the same level in $23 \%$ of investigated sides and at an anterior level to it in $23 \%$ of them. The most extreme result to that of the present study has been shown by Lee ${ }^{(14)}$ who found that the average coronal distance from SSO to PWMS measured radiologically by CT was $0.78 \mathrm{~mm}$. He also found that the SSO was behind, at the same coronal level with and in front of the PWMS in $44.4 \%, 29.3 \%$ and $26.3 \%$ of studied sides respectively. Variations in the results among these studies that evaluated the distance between the PWMS and the SSO including ours may be explained by one or more of the following possible reasons.

First, calculation methodology: Lee ${ }^{(14)}$ used relative, not absolute, values for the depth differences between the two sites during calculation of the mean so negative values were given for differences when the SSO is in front of the PWMS and this can explain the very small calculated average in his study. Second, measurement technique: As previously mentioned, some studies used CT to measure the distance between the SSO and PWMS while others measured it directly through the nasal cavity either intraoperatively or in cadavers. Third, racial variation: Differences among races may be also another explanation as our study and that of Albahkaly $\boldsymbol{e t} \boldsymbol{a l}{ }^{(\mathbf{1 2})}$ were done in the Middle East, the studies of Casiano ${ }^{(\mathbf{6})}$ and Dedhia et al. ${ }^{(\mathbf{1 3 )})}$ were done at USA while patients of Lee ${ }^{(14)}$ study were Korean.

Beside the relatively narrow range of the distance between the PWMS and the SSO shown by this study, the consistency of this wall, its ease to be found and the common to be exposed during surgery makes it 
an ideal anatomical reference to identify the SSO according to the definition described by Casiano $^{(\mathbf{6})}$.

To our knowledge, our study is the first one to investigate the correlation between preoperative $\mathrm{CT}$ and intraoperative technique as methods of measurement of the distance between the SSO and the PWMS in the same patients. We found good agreement between both techniques $(r=0.864)$ with no significant difference between the mean distance measured by CT and that measured during surgery $(\mathrm{p}=0.246)$. Such finding indicates that preoperative CT could be used to preoperatively determine the approximate distance between the SSO and the PWMS for each individual patient. This may help the surgeon to have more safe localization of the SSO and entry of the sphenoid sinus.

The relatively small number of studied sides is a potential limitation to our study. This could be explained by the multiple exclusion criteria and the need of middle meatal antrostomy and sphenoid sinusotomy at the same side to be included in the study. More similar studies with bigger sample size are recommended to give more strength to our findings.

\section{CONCLUSION}

The PWMS may be utilized as an ideal landmark to locate the SSO during endoscopic surgery. Preoperative CT could be a reliable tool to individually determine the difference in depth between the SSO and the PWMS.

\section{Financial support and sponsorship: Nil.}

\section{Conflict of interest: Nil.}

\section{REFERENCES}

1. Levine H, Clemente M (2005): Surgical Anatomy of the Paranasal Sinus in Sinus Surgery: Endoscopic and Microscopic Approaches. New York, NY: Thieme: Pp. 13-33. http://vlib.kmu.ac.ir/kmu/handle/kmu/92139

2. Yilmaz N, Kose E, Dedeoglu N et al. (2016): Detailed anatomical analysis of the sphenoid sinus by cone-beam computed tomography. J Craniofac Surg., 27(6):549-552.
3. Abuzayed B, Tanrio"ver N, Ozlen F et al. (2009): Endoscopic endonasal transsphenoidal approach to the sellar region: results of endoscopic dissection on 30 cadavers. Turk Neurosurg., 19(3):237-244.

4. Locatelli M, Caroli M, Pluderi M et al. (2011): Endoscopic transsphenoidal optic nerve decompression: an anatomical study. Surg Radiol Anat., 33(3):257-262.

5. Gupta T, Aggarwal A, Sahni D (2013): Anatomical landmarks for locating the sphenoid ostium during endoscopic endonasal approach: a cadaveric study. Surg Radiol Anat., 35(2):137-142.

6. Casiano R (2001): A stepwise surgical technique using the medial orbital floor as the key landmark in performing endoscopic sinus surgery. The Laryngoscope, 111(6): 964-974.

7. Bolger W, Keyes A, Lanza D (1999): Use of the superior meatus and superior turbinate in the endoscopic approach to the sphenoid sinus. Otolaryngol Head Neck Surg., 120(3): 308-313.

8. Hidir Y, Battal B, Durmaz A et al. (2011): Optimum height from the roof of the choana for seeking the sphenoid ostium. J Craniofac Surg., 22(3):1077-9.

9. Turgut S, Gumusalan Y, Arifoglu Y et al. (1996): Endoscopic anatomic distances on the lateral nasal wall. J Otolaryngol., 25:371-374.

10. Ecevit M, Zeybek G, Kiray A et al. (2015): Sphenovomerine suture: a useful landmark for locating sphenoid sinus ostium. J Craniofac Surg., 26(1):264-267.

11. Halawi A, Simon P, Lidder A et al. (2015): The relationship of the natural sphenoid ostium to the skull base. Laryngoscope, 125(1):75-79.

12. Albahkaly S, Alqahtani S, Aldajani $\mathrm{N}$ et al. (2018): Utility of sphenoid ostium in relation to posterior wall of maxillary sinus in CT scan. Australasian Medical Journal, 11(9): 448-452.

13. Dedhia R, Hsieh T, Rubalcava Y et al. (2019): Posterior maxillary sinus wall: A landmark for identifying the sphenoid sinus ostium. Ann Otol Rhinol Laryngol., 128(3):215-219.

14. Lee $\mathbf{S}$ (2017): The relationship of the medial roof and the posterior wall of the maxillary sinus to the sphenoid sinus: a radiologic study. Braz $\mathrm{J}$ of Otorhinolaryngol., 83(4): 375-380. 